Review Article

\title{
Legionnaires Disease
}

\section{Sandeep K.R. ${ }^{1}$, Sandhya Rani B.S. ${ }^{2}$}

${ }^{1}$ Asso.Professor, Department of Community Health Nursing, ${ }^{2}$ Asst.Professor, Department of Medical Surgical Nursing, Ganga Sheel School of Nursing, Bareilly, U.P.

*Corresponding Author : Sandeep K.R., Associate Professor, Department Of Community Health Nursing, Ganga Sheel School of Nursing, Bareilly, U.P. E-mail : sbgsandeep@gmail.com

Received

: 17.01 .2018

Review Completed : 18.04.2018

Accepted

25.04 .2018

Keywords : Legionella bacteria, Relative bradycardia, Pontiac fever, pneumonia

\begin{tabular}{|c|}
\hline Access this article online \\
\hline Quick Response Code \\
\hline
\end{tabular}

\begin{abstract}
Legionnaires' disease is a form of atypical pneumonia caused by any type of Legionella bacteria. The bacterium is found naturally in fresh water.[4] It can contaminate hot water tanks, hot tubs, and cooling towers of large air conditioners.[4] It is usually spread by breathing in mist that contains the bacteria.[4] It can also occur when contaminated water is aspirated.[4] It typically does not spread directly between people and most people who are exposed do not become infected.[4] Risk factors for infection include older age, history of smoking, chronic lung disease, and poor immune function.[5] There is still a low level of clinical awareness regarding Legionnaires' disease 25 years after it was first detected.
\end{abstract}

\section{Definition}

Legionnaires' disease is a form of atypical pneumonia caused by any type of Legionella bacteria.[3] Signs and symptoms include cough, shortness of breath, high fever, muscle pains, and headaches.[2] Nausea, vomiting, and diarrhea may also occur.[1] This often begins two to ten days after exposure.[2]

\section{Incidence}

The prevalence of Legionella pneumophila causing community-acquired pneumonia (CAP) in patients who were admitted to hospital was investigated. Between April 1997 and December 1998, 60 patients admitted to the All India Institute of M edical Sciences with CAP, were included in the study Between J une 2015 and January 2016, 87 cases of Legionnaires' disease were reported by the Michigan Department of Health and Human Services for the city of Flint, Michigan and surrounding areas. 10 of those cases were fatal.[20]In November 2017, an outbreak was detected at Hospital de São Francisco Xavier, Lisbon,
Portugal, with up to 53 people being diagnosed with the disease and five of them dying from it.[21]

Causes: Over $90 \%$ of cases of Legionnaires' disease are caused by the bacteria Legionella pneumophila. Other types include L. longbeachae, L. feeleii, L. micdadei, and L. anisa

\section{Signs and symptoms}

The length of time between exposure to the bacteria and the appearance of symptoms is generally two to ten days, but can rarely extend to as much as 20 days.[6] For the general population, among those exposed between 0.1 and $5 \%$ develop disease, while among those in hospital between 0.4 and $14 \%$ develop disease.[6]

Those with Legionnaires' disease usually have fever, chills, and a cough, which may be dry or may produce sputum. Almost all with Legionnaires' experience fever, while approximately half have cough with sputum, and one third cough up blood or bloody sputum. Some also have muscle 
aches, headache, tiredness, loss of appetite, loss of coordination (ataxia), chest pain, or diarrhea and vomiting.[7] Up to half of those with Legionnaires' have gastrointestinal symptoms, and almost half have neurological symptoms,[6] including confusion and impaired cognition.[8] "Relative bradycardia" may also be present, which is low or low-normal heart rate despite the presence of a fever.

Laboratory tests may show that kidney functions, liver functions and electrolyte levels are abnormal, which may include low sodium in the blood. Chest X-rays often show pneumonia with consolidation in the bottom portion of both lungs. It is difficult to distinguish Legionnaires' disease from other types of pneumonia by symptoms or radiologic findings alone; other tests are required for definitive diagnosis.

Persons with Pontiac fever experience and muscle aches without pneumonia generally recover in two to five days without treatment. For Pontiac fever the time between exposure and symptoms is generally a few hours to two days.

Transmission: Legionnaires' disease is usually spread by the breathing in of aerosolized water and/or soil contaminated with the Legionella bacteria.[7] Experts have stated that Legionnaires' disease is not transmitted from person to person. Rarely, it has been transmitted by direct contact between contaminated water and surgical wounds.[7] The bacteria grow best at warm temperatures.[4] It thrives at water temperatures between 25 and $45^{\circ} \mathrm{C}\left(77\right.$ and $\left.113^{\circ} \mathrm{F}\right)$, with an optimum temperature of $35^{\circ} \mathrm{C}\left(95^{\circ} \mathrm{F}\right)$.[21] Temperatures above $60^{\circ} \mathrm{C}\left(140{ }^{\circ} \mathrm{F}\right)$ kill it.[11] Sources where temperatures allow the bacteria to thrive include hot water tanks, cooling towers, and evaporative condensers of large air conditioning systems, such as those commonly found in hotels and large office buildings.[12] Though the first known outbreak was in Philadelphia, Pennsylvania, cases of legionellosis have occurred throughout the world.[6] The disease may also be transmitted from contaminated aerosols generated in hot tubs if the disinfection and maintenance program is not followed rigorously.[14] Freshwater ponds, creeks, and ornamental fountains are potential sources of Legionella.[15] The disease is particularly associated with hotels, fountains, cruise ships, and hospitals with complex potable water systems and cooling systems. L. pneumophila thrives in aquatic systems where it is established within amoebae in a symbiotic relationship.[13] Legionella bacteria survive in water as intracellular parasites of water-dwelling protozoae, such as amoebae. Amoebae are often part of bio-films, and once Legionella and infected amoebae are protected within a bio-film, they are particularly difficult to destroy.[7]

\section{Mechanism}

Legionella enters the lung either by aspiration of contaminated water or inhalation of aerosolized contaminated water or soil. In the lung, the bacteria are consumed by macrophages, a type of white blood cell, inside of which the Legionella bacteria multiply causing the death of the macrophage. Once the macrophage dies, the bacteria are released from the dead cell to infect other macrophages. Virulent strains of Legionella kill macrophages by blocking the fusion of phagosomes with lysosomes inside the host cell; normally the bacteria are contained inside the phagosome, which merges with a lysosome, allowing enzymes and other chemicals to break down the invading bacteria.[6]

Diagnosis: The most useful diagnostic tests detect the bacteria in coughed up mucus, find Legionella antigens in urine samples, or allow comparison of Legionella antibody levels in two blood samples taken 3 to 6 weeks apart. A urine antigen test is simple, quick, and very reliable, but it will only detect Legionella pneumophila serogroup 1 , which accounts for 70 percent of disease caused by $L$. pneumophila, which means use of the urine antigen test alone may miss as many as 30\% of cases.[10] This test was developed by Richard Kohler in 1982.[16] When dealing with Legionella pneumophila serogroup 1, the urine antigen test is useful for early detection of Legionnaire's disease and initiation of treatment, and has been helpful in early detection of outbreaks. However, it will not identify 
the specific subtypes, so it cannot be used to match the person with the environmental source of infection. The Legionella bacteria can be cultured from sputum or other respiratory samples. Legionella stains poorly with Gram stain, stains positive with silver, and is cultured on charcoal yeast extract with iron and cysteine (CYEagar).

A significant under-reporting problem occurs with legionellosis. Even in countries with effective health services and readily available diagnostic testing, about 90 percent of cases of Legionnaires' disease are missed. This is partly due to Legionnaires' disease being a relatively rare form of pneumonia, which many clinicians may not have encountered before and thus may misdiagnose. A further issue is that people with legionellosis can present with a wide range of symptoms, some of which (such as diarrhea) may distract clinicians from making a correct diagnosis. [17]

\section{Prevention}

Although the risk of Legionnaires' disease being spread by large-scale water systems cannot be eliminated, it can be greatly reduced by writing and enforcing a highly detailed, systematic water safety plan appropriate for the specific type of facility involved (office building, hospital, hotel, spa, cruise ship, etc.)[6] Some of the elements that such a plan may include are the following:

- Keeping water temperature either above or below the 20-50 ${ }^{\circ} \mathrm{C}\left(68-122{ }^{\circ} \mathrm{F}\right)$ range in which the Legionella bacterium thrives.

- Preventing stagnation, for example by removing from a network of pipes any sections that have no outlet (dead ends). Where stagnation is unavoidable, for example when a wing of a hotel is closed for the off-season, systems must be thoroughly disinfected just prior to resuming normal operation.

- Preventing the build up of bio-film, for example by not using (or by replacing) construction materials that encourage its development, and by reducing the quantity of nutrients for bacterial growth that enter the system.

- Periodic disinfection of the system, by high heat or a chemical biocide, and the use of chlorination where appropriate. There is evidence that treatment of water with copper-silver ionization or ultraviolet light may also be effective.[32]

- System design (or renovation) that reduces the production of aerosols and reduces human exposure to them, for example by directing them well away from building air intakes.

An effective water safety plan will also cover such matters as training, record-keeping, communication among staff, contingency plans and management responsibilities. The format and content of the plan may be prescribed by public health laws or regulations.[6]

\section{Treatment}

Effective antibiotics include most macrolides, tetracyclines, ketolides, and quinolones.[7] Legionella multiply within the cell, so any effective treatment must have excellent intracellular penetration. Current treatments of choice are the respiratory tract quinolones (levofloxacin, moxifloxacin, gemifloxacin) or newer macrolides (azithromycin, clarithromycin, roxithromycin). The antibiotics used most frequently have been levofloxacin, doxycycline, and azithromycin.

M acrolides (azithromycin) are used in all age groups, while tetracyclines (doxycycline) are prescribed for children above the age of 12 and quinolones (levofloxacin) above the age of 18. Rifampicin can be used in combination with a quinolone or macrolide. It is uncertain whether rifampicin is an effective antibiotic to take for treatment. The Infectious Diseases Society of America does not recommend the use of rifampicin with added regimens. Tetracyclines and erythromycin led to improved outcomes compared to other antibiotics in the original American Legion outbreak. These antibiotics are effective because they have excellent intracellular penetration in Legionellainfected cells. The recommended treatment is 5- 10 days of levofloxacin or 3-5 days of azithromycin, but in people who are immune compromised, have severe disease, or other pre-existing health conditions, longer antibiotic use may be necessary.[7] During outbreaks, prophylactic antibiotics have been successfully used to prevent Legionnaires' 
disease in high-risk individuals who have possibly been exposed.[7]

The mortality at the original American Legion convention in 1976 was high (29 deaths in 182 infected individuals[33]) because the antibiotics used (including penicillins, cephalosporins, and aminoglycosides) had poor intracellular penetration. Mortality has plunged to less than $5 \%$ if therapy is started quickly. Delay in giving the appropriate antibiotic leads to higher mortality.

\section{References}

1. Cunha, BA; Burillo, A; Bouza, E (23 January 2016). "Legionnaires' disease". Lancet. 387 (10016): 376-85.

2. Cunha, BA; Burillo, A; Bouza, E (23 January 2016). "Legionnaires' disease". Lancet. 387 (10016): 376-85.

3. "Legionella (Legionnaires' Disease and Pontiac Fever) About the Disease". CDC. January 26, 2016. Archived from the original on 25 M arch 2016. Retrieved 21 M arch 2016.

4. "Legionella (Legionnaires' Disease and Pontiac Fever) Causes and Transmission". CDC. March 9, 2016. Archived from the original on 25 March 2016. Retrieved 21 M arch 2016.

5. "Legionella (Legionnaires' Disease and Pontiac Fever) People at Risk". CDC. January 26, 2016. Archived from the original on 27 M arch 2016. Retrieved $21 \mathrm{M}$ arch 2016.

6. Legionella and the prevention of legionellosis (PDF). Geneva: World Health Organization. 2007. ISBN 9241562978. Archived (PDF) from the original on 2011-05-03.

7. Cunha, Burke A; Burillo, Almudena; Bouza, Emilio (2015). "Legionnaires' disease". The Lancet. 387: 376-85. doi:10.1016/S01406736(15)60078-2. ISSN 0140-6736. PM ID 26231463.

8. Edelstein PH. Legionnaires Disease: History and clinical findings. Open Access Biology "Archived copy". Archived from the original on 201006-08. Retrieved 2010-06-26.

9. Ostergaard L, Huniche B, Andersen PL (November 1996). "Relative bradycardia in infectious diseases". J. Infect. 33 (3): 185-91.

10. Fields, B. S.; Benson, R. F.; Besser, R. E. (2002). "Legionella and Legionnaires' Disease: 25 Years of Investigation". Clinical M icrobiology Reviews. 15 (3): 506-526.

11. "HSE Legionnaires' disease Hot and cold water systems Things to consider". Archived from the original on 2016-03-29.

12. van Heijnsbergen, Eri; Schalk, Johanna A. C.; Euser, Sjoerd M.; Brandsema, Petra S.; den Boer, Jeroen W.; de Roda Husman, Ana M aria (2015). "Confirmed and Potential Sources of Legionella Reviewed". Environmental Science \& Technology. 49 (8): 4797-4815.

13. Winiecka-Krusnell J, Linder E (1999). "Free-living amoebae protecting Legionella in water: The tip of an iceberg?". Scandinavian journal of infectious diseases. 31 (4): 383-385.

\section{Prognosis}

The fatality rate of Legionnaires' disease has ranged from $5 \%$ to $30 \%$ during various outbreaks and approaches $50 \%$ for nosocomial infections, especially when treatment with antibiotics is delayed.[22] Hospital-acquired Legionella pneumonia has a fatality rate of $28 \%$, and the principal source of infection in such cases is the drinking-water distribution System.[23]

14. Silivanch v. Celebrity Cruises, Inc., 171 F.Supp.2d 241 (S.D.N.Y. 2001) (plaintiff successfully sued cruise line and manufacturer of filter after catching disease on cruise)

15. Winn WC Jr (1996). "Legionella". In Baron S; et al. Baron's Medical Microbiology (4th ed.). Univ of Texas M edical Branch. ISBN 0-96311721-1. Via NCBI Bookshelf.

16. Kohler R, Wheat LJ (September 1982). "Rapid diagnosis of pneumonia due to Legionella pneumophila serogroup 1". J. Infect. Dis. 146 (3):

17. M akin, T (January 2008). "Legionella bacteria and solar pre-heating of water for domestic purposes" (PDF). UK Water Regulations Advisory Scheme Report: 4.

18. Almeida, D; Cristovam, E; Caldeira, D; Ferreira, J); Marques, T (1 November 2016). "Are there effective interventions to prevent hospital-acquired Legionnaires' disease or to reduce environmental reservoirs of Legionella in hospitals? A systematic review". American journal of infection control. 44 (11): e183-e188.

19. Winn WC (1988). "Legionnaires disease: historical perspective". Clinical Microbiology Reviews. 1 (1): 60-81.

20. Sarah Kaplan (14 January 2016). "Flint, Mich., has 10 fatal cases of Legionnaires' disease; unclear if linked to water". Washington Post. Archived from the original on $28 \mathrm{M}$ arch 2016. Retrieved $31 \mathrm{March}$ 2016.

21. http://rr.sapo.pt/noticia/ 98404/legionella-numero-de-casos-sobepara-53

22. "Legionnaire disease". M edline Plus. US National Library of M edicine. Archived from the original on 5July 2015. Retrieved 5 August 2015.

23. Stout JE, M uder RR, M ietzner S, Wagener M M, Perri MB, DeRoos K, Goodrich D, Arnold W, Williamson T, Ruark O, Treadway C, Eckstein EC, M arshall D, Rafferty M E, Sarro K, Page J, Jenkins R, Oda G, Shimoda KJ, Zervos MJ, Bittner M, Camhi SL, Panwalker AP, Donskey CJ, Nguyen MH, Holodniy M, Yu VL (July 2007). "Role of environmental surveillance in determining the risk of hospital-acquired legionellosis: a national surveillance study with clinical correlations". Infect Control Hosp Epidemiol. 28 (7): 818-24 